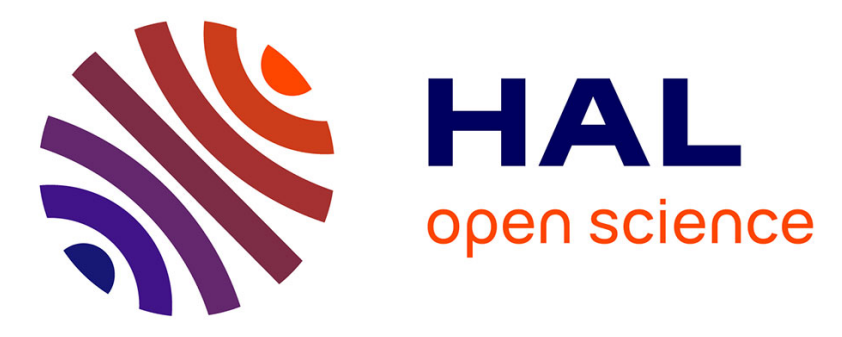

\title{
Use of infrared thermography for the study of evaporation in a square capillary tube
}

Fabien Chauvet, Sébastien Cazin, Paul Duru, Marc Prat

\section{To cite this version:}

Fabien Chauvet, Sébastien Cazin, Paul Duru, Marc Prat. Use of infrared thermography for the study of evaporation in a square capillary tube. International Journal of Heat and Mass Transfer, 2010, 53 (9-10), pp.1808-1818. 10.1016/j.ijheatmasstransfer.2010.01.008 . hal-01360010

\section{HAL Id: hal-01360010 https://hal.science/hal-01360010}

Submitted on 5 Sep 2016

HAL is a multi-disciplinary open access archive for the deposit and dissemination of scientific research documents, whether they are published or not. The documents may come from teaching and research institutions in France or abroad, or from public or private research centers.
L'archive ouverte pluridisciplinaire HAL, est destinée au dépôt et à la diffusion de documents scientifiques de niveau recherche, publiés ou non, émanant des établissements d'enseignement et de recherche français ou étrangers, des laboratoires publics ou privés. 


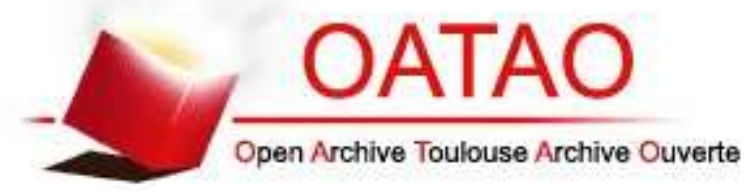

\section{Open Archive TOULOUSE Archive Ouverte (OATAO)}

OATAO is an open access repository that collects the work of Toulouse researchers and makes it freely available over the web where possible.

This is an author-deposited version published in : http://oatao.univ-toulouse.fr/ Eprints ID : 16044

To link to this article : DOI : $10.1016 / \mathrm{j}$. .ijheatmasstransfer.2010.01.008 URL : http://dx.doi.org/10.1016/j.ijheatmasstransfer.2010.01.008

To cite this version : Chauvet, Fabien and Cazin, Sébastien and Duru, Paul and Prat, Marc Use of infrared thermography for the study of evaporation in a square capillary tube. (2010) International Journal of Heat and Mass Transfer, vol. 53 ( $\mathrm{n}^{\circ}$ 9-10). pp. 18081818. ISSN 0017-9310

Any correspondence concerning this service should be sent to the repository administrator: staff-oatao@ listes-diff.inp-toulouse.fr 


\title{
Use of infrared thermography for the study of evaporation in a square capillary tube
}

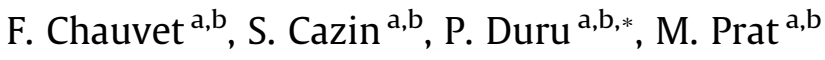 \\ a Université de Toulouse, INPT, UPS, IMFT (Institut de Mécanique des Fluides de Toulouse), Allée Camille Soula, F-31400 Toulouse, France \\ ${ }^{\mathrm{b}}$ CNRS, IMFT, F-31400 Toulouse, France
}

Keywords:

Evaporation

Capillary tube

Liquid film

Corner flow

Infrared thermography

\begin{abstract}
A B S T R A C T
In this paper we report experimental results on evaporation of a volatile wetting liquid in a capillary tube of square internal cross section, when conditions are such that liquid films develop along the tube internal corners under the effect of capillary forces, as the bulk meniscus recedes inside the tube. Combining an infrared thermography technique with visualizations by ombroscopy makes it possible to determine the time-space evolution of the temperature minimum on the capillary outer surface together with the bulk meniscus position within the tube. When the tube is held horizontal, the temperature minimum stays at the tube entrance and the evaporation rate reaches a stationary value. In contrast with the horizontal case, the position of the temperature minimum changes when the bulk meniscus has sufficiently receded inside the tube when the tube is vertical and opened at the top. The rate of evaporation then decreases significantly. This is explained by the thinning of the corner films in the vertical tube entrance region, under the conjugated effects of gravity and viscous forces up to the depinning of the films from the tube entrance. When the tube is held horizontal, the capillary effects are dominant and the film thickness remains essentially constant in the tube entrance region. This analysis is supported by a simple model of liquid flow within the corner films.
\end{abstract}

\section{Introduction}

Evaporation from a meniscus plays an important role in many applications such as capillary pumped loops, heat pipes, fuel cells and drying of porous media. In this context, the study of evaporation of a liquid confined in a single microchannel can be regarded as a first step before more complex situations such as, for example, networks of interconnected capillaries [1]. In recent years, evaporation driven by mass transfer has been studied experimentally in microchannels of rectangular cross section [2], as well as in capillary tubes of square cross section [3]. All these works, see also [4], indicate that evaporation in a channel with corners is much faster than in a channel of circular cross section. This is attributed to the effects of "thick" liquid films trapped along the channel corners under the action of capillary effects. Because of these films, modelling of evaporation in a channel of polygonal cross section is significantly more involved than for the classical circular tube. A model of liquid flow with evaporation in a channel of square cross section was presented in [5] in relation with the modelling of drying of porous media. Depending on the competition between the capillary, viscous and gravity forces, various evaporation regimes can

\footnotetext{
* Corresponding author. Address: Université de Toulouse, INPT, UPS, IMFT (Institut de Mécanique des Fluides de Toulouse), Allée Camille Soula, F-31400 Toulouse, France. Tel.: +33 5612858 77; fax: +33 561285899 .

E-mail address: duru@imft.fr (P. Duru).
}

be distinguished. The regime dominated by the capillary forces was mainly considered in [6], whereas a modelling of other regimes was presented in [7]. Tubes of triangular or hexagonal cross sections have been considered as well, see [8] where first results on the influence of contact angle are also presented. However, there is a lack of experimental data and, consequently, of quantitative comparisons between the available models and experimental data. In this context, the general objective of the present work is to contribute to fill this gap by combining careful experimental studies with a proper modelling. First results in this direction were reported in [9] but only for a tube in vertical position. Here we consider also the case of a tube in a horizontal position and use the IR thermography technique to analyze in more details the evaporation process. A preliminary version of the present paper was presented in [10]. Here much more details are given, together with additional experimental results.

More specifically, we study evaporation in capillary tubes of square or circular cross section, the internal side length of which is $1 \mathrm{~mm}$, a value lower than the capillary length. As discussed in several previous works (see e.g. [8] and references therein) and sketched in Fig. 1, thick liquid films can be trapped by capillarity along the four internal corners of a square tube as the bulk meniscus recedes inside the tube under the effect of evaporation. These corner films provide paths for the liquid between the receding bulk meniscus and the entrance of the tube. The liquid is transported within the films under the action of the pressure gradient induced 


\begin{tabular}{|c|c|c|c|}
\hline \multicolumn{4}{|c|}{ Nomenclature } \\
\hline$A$ & gain of a pixel of the infrared camera detector & $P_{\infty}$ & partial pressure of hexane at the tube outlet, $\mathrm{Nm}^{-2}$ \\
\hline$A_{c}$ & liquid film cross section area, $\mathrm{m}^{2}$ & $R$ & curvature radius of the corner film, $\mathrm{m}$ \\
\hline$A_{i}$ & gain of pixel $i$ of the infrared camera detector & $R^{\prime}$ & dimensionless curvature radius of the corner film \\
\hline$B$ & offset of a pixel of the infrared camera detector & $t$ & time, $s$ \\
\hline$B_{i}$ & offset of pixel $i$ of the infrared camera detector & $T$ & surface temperature of the imaged object, $\mathrm{K}$ \\
\hline Bo & Bond number & $T_{a}$ & ambient temperature, $\mathrm{K}$ \\
\hline $\mathrm{Ca}$ & capillary number & $T_{b}$ & black body temperature, $\mathrm{K}$ \\
\hline$d$ & capillary tube internal side length, $\mathrm{m}$ & $T_{c}$ & infrared camera temperature, $\mathrm{K}$ \\
\hline$D$ & diffusion coefficient of hexane in air, $\mathrm{cm}^{2} \mathrm{~s}^{-1}$ & $T_{\min }$ & minimum tube wall temperature, $\mathrm{K}$ \\
\hline$D L^{0}$ & "effective" emittance of the black body, in digital levels & $z$ & coordinate, m \\
\hline$D L_{m}$ & signal received by a pixel of the infrared camera & $z_{0}$ & bulk meniscus position, $\mathrm{m}$ \\
\hline$D L_{m, i}$ & $\begin{array}{l}\text { detector, in digital levels } \\
\text { signal received by pixel } i \text { of the infrared camera } \\
\text { detector, in digital levels }\end{array}$ & $\begin{array}{l}z_{f} \\
z_{\min } \\
\beta\end{array}$ & $\begin{array}{l}\text { film tip location, } m \\
\text { minimum temperature location along the tube wall, } \mathrm{m} \\
\text { dimensionless hydraulic resistance for corner flow }\end{array}$ \\
\hline$D L_{u w, \text { tube }}$ & $\begin{array}{l}\text { corrective term for infrared measurement, in digital } \\
\text { levels }\end{array}$ & $\epsilon$ & $\begin{array}{l}\text { emissivity of the imaged surface } \\
\text { emissivity of the black body }\end{array}$ \\
\hline $\begin{array}{l}e \\
f(T)\end{array}$ & $\begin{array}{l}\text { thickness of the liquid film, } m \\
\text { calibration function for a pixel of the infrared camera }\end{array}$ & $\phi_{m}$ & $\begin{array}{l}\text { radiative flux received by the detector of the IR camera, } \\
W \mathrm{~m}^{-2}\end{array}$ \\
\hline $\begin{array}{l}F \\
g\end{array}$ & $\begin{array}{l}\text { mathematical function } \\
\text { gravity acceleration, } \mathrm{ms}^{-2}\end{array}$ & $\phi_{u w}$ & $\begin{array}{l}\text { "unwanted" radiative flux received by the detector of } \\
\text { the IR camera, } \mathrm{Wm}^{-2}\end{array}$ \\
\hline$g\left(T_{b}\right)$ & $\begin{array}{l}\text { black body calibration function for a pixel of the } \\
\text { infrared camera }\end{array}$ & $\begin{array}{l}\gamma \\
\lambda\end{array}$ & $\begin{array}{l}\text { surface tension, } \mathrm{Nm}^{-1} \\
\text { geometrical factor }\end{array}$ \\
\hline$k_{b}$ & Boltzmann constant, $\mathrm{JK}^{-1}$ & $\mu_{l}$ & liquid viscosity, $\mathrm{kg} \mathrm{m}^{-1} \mathrm{~s}^{-1}$ \\
\hline$M$ & hexane molecular weight, $\mathrm{g} \mathrm{mol}^{-1}$ & $\theta$ & equilibrium contact angle \\
\hline$N_{a}$ & Avogadro constant, $\mathrm{mol}^{-1}$ & $\rho_{l}$ & density of liquid, $\mathrm{kg} \mathrm{m}^{-3}$ \\
\hline$p_{l}$ & pressure in the corner film, $\mathrm{Nm}^{-2}$ & $\tau_{c}$ & characteristic time for evaporation, s \\
\hline$P_{a}$ & atmospheric pressure, $\mathrm{N} \mathrm{m}^{-2}$ & $\tau_{d}$ & characteristic time for heat diffusion within the tube \\
\hline$P_{s}$ & $\begin{array}{l}\text { saturation partial pressure of hexane at ambient } \\
\text { pressure and temperature, } \mathrm{Nm}^{-2}\end{array}$ & & wall, s \\
\hline
\end{tabular}

by the meniscus curvature variation along the films. This effect is therefore termed capillary pumping. As a result, the phase change occurs preferentially at the entrance of the tube as long as the corner liquid films remain attached at the tube entrance. This very efficient transport mechanism of liquid by the films is naturally absent in a circular tube, in which the transport mechanism between the receding bulk meniscus and the tube entrance is the poorly efficient molecular diffusion in the gas phase (this case is usually referred to as the "Stefan tube" problem [11]). This explains why evaporation can be several orders of magnitude faster in a tube with corners compared to a circular tube (see [8]). Adsorbed thin films may also be present over the internal surfaces of the tube not covered by the thick films. However, the hydraulic conductivity of the adsorbed films is very small compared to that of the thick

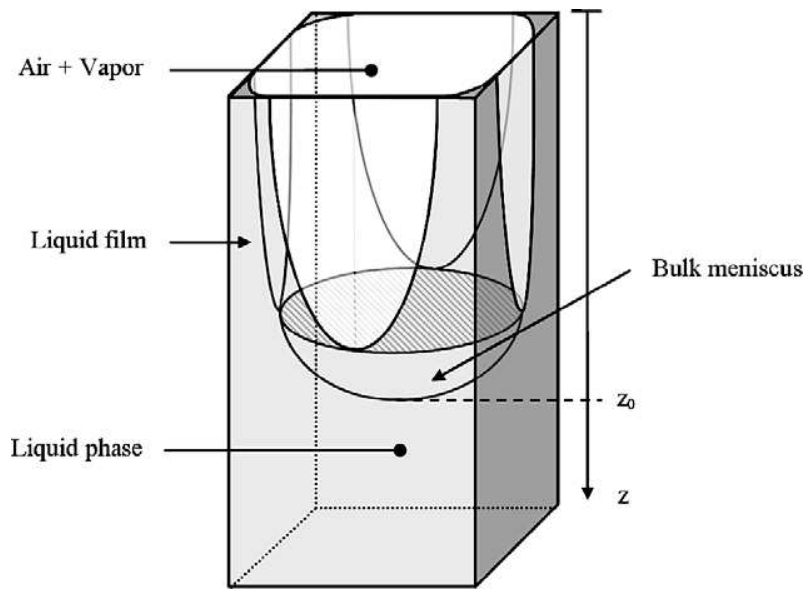

Fig. 1. Sketch of the thick liquid films in a capillary tube of square cross section. films in tubes of internal side length much greater than the typical thickness of adsorbed films (a few nanometers), as considered here. Hence the effect of the adsorbed films can be neglected in the present case. As discussed in [8], the wetting contact angle of the liquid must be sufficiently low for the corner films to develop. The value of the contact angle below which the corner films can exist depends on the corner internal angle. For a tube of square cross section (corners internal angle $=90^{\circ}$ ), the liquid contact angle $\theta$ must be less than $45^{\circ}$. The results presented in this paper have been obtained for evaporation of hexane in borosilicate glass tubes, i.e. for an almost perfectly wetting liquid $\left(\theta \approx 0^{\circ}\right)$. Evaporation is self-induced in a stagnant air atmosphere at ambient temperature and atmospheric pressure. Hence, there is no external forced convective flow imposed near the tube entrance.

The paper is organized as follows. The experimental set-up and techniques are presented first. An ombroscopy visualization technique is used to track the volume of liquid contained in the capillary during evaporation and thus to measure the evaporation rate. A distinguishing feature of the present work is to couple this rather standard ombroscopy technique with an infrared (IR) thermography technique. The use of the IR technique in the present context is detailed in the third section of the paper. Then, in Section 4, we present some representative results illustrating the interest of combining the two types of visualizations for analyzing evaporation in capillary tubes. The paper ends with an analysis of the results using a simple model of liquid flows within the corner films.

Before going into the details of the present work, it is interesting to notice that an IR technique has already been used for studying evaporation in capillary tubes. In [12], IR measurements of temperature along the meniscus interface of volatile liquids in capillary tubes of circular cross section were reported. Interestingly, these measurements show that the temperature is not uniform along the meniscus interface and lower at the meniscus triple line where 
the evaporation rate is greater than in the middle of the meniscus. Hence the IR technique was shown to be a valuable tool for detecting the temperature sink effect associated with evaporation, a feature we also use in the present study. However, the results reported in [12] were for circular tubes (as opposed to square tubes in the present case) and for a stationary meniscus located at the tube entrance (as opposed to receding ones in the present case).

\section{Experimental set-up}

The $10 \mathrm{~cm}$ long square capillary tubes used in the present study are made of borosilicate glass: the internal side length and the wall thickness of the tubes are $1 \mathrm{~mm}$ and $0.2 \mathrm{~mm}$ respectively (Vitrocom). A tube with a circular cross section (internal diameter $1 \mathrm{~mm}$, wall thickness $0.2 \mathrm{~mm}$ ) is also used as a reference case for some experiments.

A capillary tube is glued by an epoxy resin directly to a syringe tip. The syringe is placed on a precision syringe pump (PHD 2000, Harvard Apparatus), allowing accurate filling of the tube by the volatile liquid (here, $n$-hexane). It is possible to position the capillary tube either vertically or horizontally, in order to assess the influence of gravity. The room temperature is controlled by an air-conditioning system. The experimental set-up is placed inside a Plexiglas enclosure which helps to further stabilize the temperature in the environment of the tube. The temperature of the air inside the enclosure is monitored by thermocouples and remains constant for the entire duration of the experiment, which can last several hours. This temperature control is crucial to avoid a possible effect of dilatation/contraction of the liquid with variations of the ambient temperature, which can distort the evaporation rate measurement.

The visualizations are done using a Phlox white LED backlight as a light source, which provides a constant and uniform light intensity and does not release heat in the strobe mode used in the present experiments. Images are acquired with a CCD camera (Sensicam, PCO). The capillary tube is placed between the light source and the camera. This ombroscopy configuration is often used to detect the location of an interface between two fluids. In the present case, the liquid and gas phases regions appear as bright on the images whereas the bulk meniscus appears as dark because light rays are deviated by the curved interface. Therefore, after binarization of the images, the position of the bulk meniscus can easily be detected (here, it is taken at the most advanced point of the gas phase into the tube, see Fig. 1). Image processing is done using the Matlab Image Processing Toolbox.

The IR thermography measurements are made using a 14-bit Focal Plane Array camera-type (Jade MWIR, Cedip) with a spectral band sensitivity ranging from 3.6 to $5.2 \mu \mathrm{m}$ and a $320 \times 240$ pixels $^{2}$ sensor cooled to $79 \mathrm{~K}$ by a Stirling engine. The IR detector integration time is set at $1 \mathrm{~ms}$. The optical axis of the infrared camera is perpendicular to the ombroscopy visualization axis. As Plexiglas is not transparent to IR radiation, an opening in one of the enclosure panels is made: it also allows the infrared camera optics to be placed close enough to the tube in order to have the necessary spatial resolution (see Section 3.2). The external side of the tube placed in front of the infrared camera has been previously painted with a spray of mat black colour. The radiative properties of the paint, in the infrared, have been estimated [13]: it is opaque in the band spectral sensitivity of the camera and its emissivity is 0.94. Therefore, reflection of the environment at ambient temperature on the capillary external surface is limited. To calibrate the camera, a black body (DCN $1000 \mathrm{~N} 4, \mathrm{HGH}$ ), consisting of a $100 \mathrm{~mm} \times 100 \mathrm{~mm}$ black surface (emissivity $\epsilon_{b}$ of 0.98 ), is used. Both cameras and light source are synchronized through a signal generator. The infrared images processing is performed using the Matlab Image Processing Toolbox.

\section{Infrared thermography}

Measuring accurately the temperature, and its spatial variations, at the outer surface of the capillary tube requires to analyse the different contributions of the radiative flux impinging on the IR camera detector when imaging the capillary tube on one hand and the black body on the other hand (during calibration). The analysis detailed below in Section 3.1 takes into account the effects of "unwanted" fluxes and permits to set-up the method used to process the IR images. Then, the exploitation of the IR results is detailed in Section 3.2 and the accuracy and limitations of this measurement are discussed.

\subsection{Infrared images processing}

The radiative flux $\Phi_{m}$ (in $\mathrm{W} \mathrm{m}^{-2}$ ) received by the detector of the IR camera can be expressed as:

$\Phi_{m}=\epsilon \phi(T)+(1-\epsilon) \phi\left(T_{a}\right)+\phi_{u w}\left(T_{a}, T_{c}\right)$.

In this expression, the absorption of radiation by air is neglected because of the short distance between the tube and the camera (always inferior to $10 \mathrm{~cm}$ ) on one hand, and the low humidity in the room in the other hand. To be rigorous, this equation should also include view factors between the radiative sources and the IR detector and a transmission coefficient to take into account radiation absorption by the camera lens. However, as their introduction do not change the results of the following discussion, they will be omitted to lighten the notations.

The first term of the right-hand side of Eq. (1) is the product of $\phi(T)$, the emittance of a black body at surface temperature $T$, and $\epsilon$ the emissivity of the observed surface. The second term in the right-hand side of Eq. (1) is the contribution of the reflection of the environment at ambient temperature $T_{a}$ on the observed surface. The last term in the right-hand side of Eq. (1) is the contribution of all "unwanted" fluxes which are due to the three following effects [14]. First, the so-called "Narcissus" effect which is caused by the reflection on the imaged object of the temperature contrast between the cooled IR detector and the hotter detector environment. The Narcissus effect generates a roughly concentric signal gradient on the IR image. For instance, when a highly reflective surface, with an uniform temperature field is observed, the signal in the centre of the obtained IR image can be significantly lower than that in the periphery [14]. Second, an illumination flux, coming from non-focussed objects present behind the object imaged. This is to be considered especially when imaging objects occupying a small portion of the viewing field (which was the case here, when working with a capillary tube). Finally, an effect produced by nonfocused radiation from "inside" the camera (including notably the radiation to the detector from the camera lenses, the temperature of which is higher than $T_{a}$, due to the heat dissipation produced by the Stirling motor). This latter effect and the Narcissus effect depend on the IR camera temperature, which is noted $T_{c}$ and is higher than $T_{a}$. It is important to note that the magnitude of these unwanted fluxes does not depend on the temperature of the imaged object, but only on the ambient temperature and on the temperature of the camera. In addition, the relative amplitudes of these effects depend on the object imaged during the present experiments. When it is the large surface (relative to the viewing field) of the black body, the illumination effect is negligible. When the imaged object is a capillary tube, the three effects are present. Note that the Narcissus effect is then more pronounced than with the black body because the emissivity of the paint is lower than that of the black body.

The camera detector responds linearly with the radiative flux $\Phi_{m}$, between the detector noise and saturation levels (the latter 
was never reached in the present experiments, given the short detector integration time used and the fact that the measured temperatures did not exceed $30^{\circ} \mathrm{C}$ ). The signal obtained at one pixel of the detector is proportional to the radiative flux received by this pixel:

$D L_{m, i}\left(T, T_{a}\right)=A_{i} \Phi_{m, i}+B_{i}$,

where $D L_{m, i}\left(T, T_{a}\right)$ is the signal given by a pixel $i$ of the IR camera detector expressed in digital levels (DL). As a focal plane array IR camera is used in the present study, each pixel has its own value for the gain $A_{i}$ and offset $B_{i}$. Because of the unwanted fluxes and of the different gain and offset values for each pixel, a perfectly uniform image (in DL) would never be obtained when imaging a surface at a perfectly uniform temperature. This becomes a problem when small temperature variations compared to the ambient temperature have to be detected as it is the case in the present study. Indeed, the amplitude of the signal of interest may be then "concealed" by the effects of the unwanted fluxes and of the IR detector non-uniformity. To get rid of this problem and be able to detect with confidence low temperature variation compared to the ambient, the following calibration procedure was used.

Using Eqs. (1) and (2), the signal obtained at a given pixel when imaging the black body can be expressed in digital levels as (the subscript $i$ will be omitted in the following):

$D L_{m}\left(T_{b}, T_{a}\right)=\epsilon_{b} D L^{0}\left(T_{b}, T_{a}\right)+\left(1-\epsilon_{b}\right) D L^{0}\left(T_{a}, T_{a}\right)$,

where $D L^{0}\left(T_{b}, T_{a}\right)=A\left(\phi\left(T_{b}\right)+\phi_{u w}\left(T_{a}, T_{c}\right)\right)+B$ is an "effective" emittance of the black body at temperature $T_{b}$, in an environment at ambient temperature $T_{a}$ and with a camera temperature $T_{c}$. The effects of unwanted fluxes present during calibration, namely the one due to the non-focussed radiation and the one due to the Narcissus effect are taken into account by including their contributions, $\phi_{u w}\left(T_{a}, T_{c}\right)$, into this effective emittance. Remarking that $D L_{m}\left(T_{a}, T_{a}\right)=D L^{0}\left(T_{a}, T_{a}\right)$, the following expression can be obtained from Eq. (3):

$D L^{0}\left(T_{b}, T_{a}\right)-D L^{0}\left(T_{a}, T_{a}\right)=\frac{1}{\epsilon_{b}}\left[D L_{m}\left(T_{b}, T_{a}\right)-D L_{m}\left(T_{a}, T_{a}\right)\right]=\frac{1}{\epsilon_{b}} g\left(T_{b}\right)$.

In practice, the measurement of $D L_{m}\left(T_{a}, T_{a}\right)$ is performed before the black body is turned on, when it is at thermal equilibrium with the environment. Then, it is turned on and several temperatures of black body are set, to measure $D L_{m}\left(T_{b}, T_{a}\right)$. The function $g\left(T_{b}\right)$ is a calibration curve for the pixel considered. A typical calibration curve is shown in Fig. 2. The temperature range used for calibration $\left(5-40^{\circ} \mathrm{C}\right)$ slightly exceeded the range of temperature obtained in the experiment $\left(15-25^{\circ} \mathrm{C}\right)$. The sensitivity of the measurement can be appreciated by noting that a $1 \mathrm{~K}$ variation in the blackbody temperature results approximately in a signal variation of 100 digital levels, in the range of temperature that is actually measured in the experiments $\left(15-25^{\circ} \mathrm{C}\right)$.

The signal obtained at the detector pixel of interest when imaging the capillary tube reads, in terms of digital levels:

$D L_{m}\left(T, T_{a}\right)=\epsilon D L^{0}\left(T, T_{a}\right)+(1-\epsilon) D L^{0}\left(T_{a}, T_{a}\right)+D L_{u w, t u b e}\left(T_{a}, T_{c}\right)$,

where $D L_{u w, t u b e}\left(T_{a}, T_{c}\right)$ is a corrective term taking into account the different contributions of the unwanted fluxes when performing a measurement on a capillary tube, compared to the measurement with the black body, namely a slightly more pronounced Narcissus effect (as the capillary tube surface has a less emissive surface, compared to that of the black body) and the presence of an illumination effect. Again, remarking that $D L_{m}\left(T_{a}, T_{a}\right)=D L^{0}\left(T_{a}, T_{a}\right)+D L_{u w, t u b e}$ $\left(T_{a}, T_{c}\right)$ and using Eq. (5) leads to:

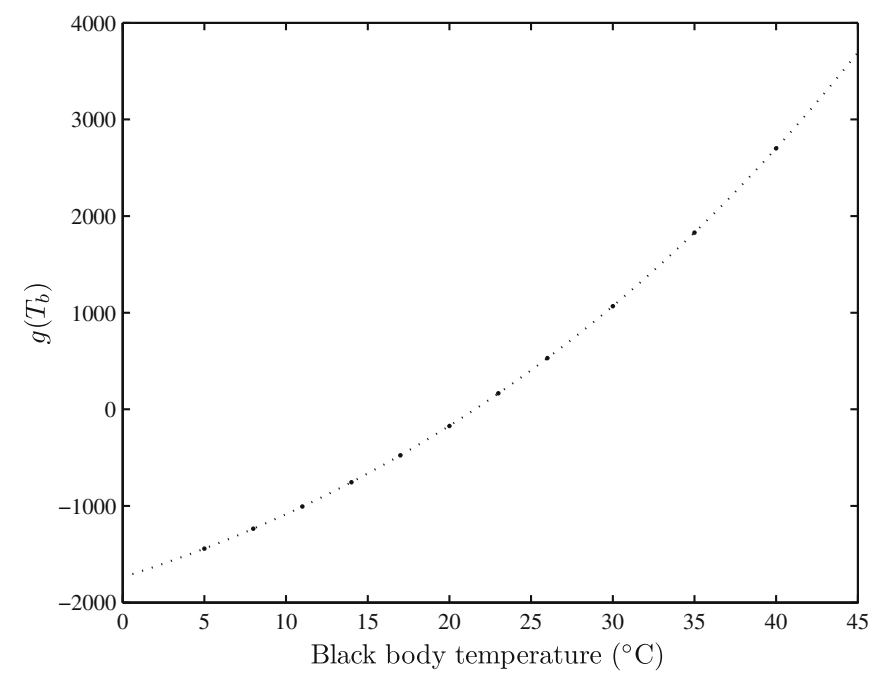

Fig. 2. A typical calibration function $g\left(T_{b}\right)$, for a given pixel of the IR detector. Circles: experimental points. Dotted line: best fitting fourth degree polynomial (least square fitting algorithm).

$D L_{m}\left(T, T_{a}\right)-D L_{m}\left(T_{a}, T_{a}\right)=\frac{\epsilon}{\epsilon_{b}} g(T)=f(T)$.

Here $D L_{m}\left(T_{a}, T_{a}\right)$ is measured by imaging the capillary tube when it is empty and at ambient temperature and $D L_{m}\left(T, T_{a}\right)$ is an image measured during the experiment. The function $f(T)$ is defined for each pixel and is obtained from the calibration curve $g(T)$ and the knowledge of both the tube paint and blackbody emissivities. It can be fitted by a fourth degree polynomial that is used to convert any measured signal in digital levels back to temperature. The above result shows that subtracting an image of the scene at ambient temperature to every IR image obtained during the experiment allows to take into account the effects of the unwanted fluxes. It is important to note that this method works if the ambient temperature is the same during experiment and calibration, and if the magnitude of the unwanted fluxes does not depend on the capillary tube temperature $T$. Using the data processing presented above, involving images subtraction and pixel by pixel calibration, is somehow similar to a data processing involving a "direct calibration" technique, see for instance [15].

\subsection{IR measurement exploitation}

Fig. 3a shows a typical "raw" IR image, i.e. without subtraction of the image at ambient temperature, obtained at the beginning of an evaporation experiment. A cooling effect due to the evaporation of hexane is detected at the top region of the tube, which appears darker on the image (lower IR signal). In Fig. 3b, the IR signal along the transverse profile shown as a line in Fig. $3 a$ (located in the cooled region) is plotted. First, it must be noted that, with the camera lens used (G1, Cedip), the tube width is imaged over roughly 30 pixels (hence the spatial resolution is approximately 21 pixels $\mathrm{mm}^{-1}$ ). Even if the slit response function of the IR system (optics and camera) was not measured in the present study, we expect that such a large spatial resolution is enough to avoid any effect of signal lowering for the signal coming from the tube wall imaged by the system [16]. In Fig. 3b, it can be seen that the signal is roughly constant in the centre of the tube (from pixel 25 to pixel 40). The two signal troughs observed at both tube edges are attributed to an effect of the tube curvature, which changes the tube apparent emissivity (the external curvature radius of the capillary tube, measured by imaging cross sections of the tube, is estimated to be roughly $250 \mu \mathrm{m})$. Therefore, the capillary tube surface temperature is con- 
(a)

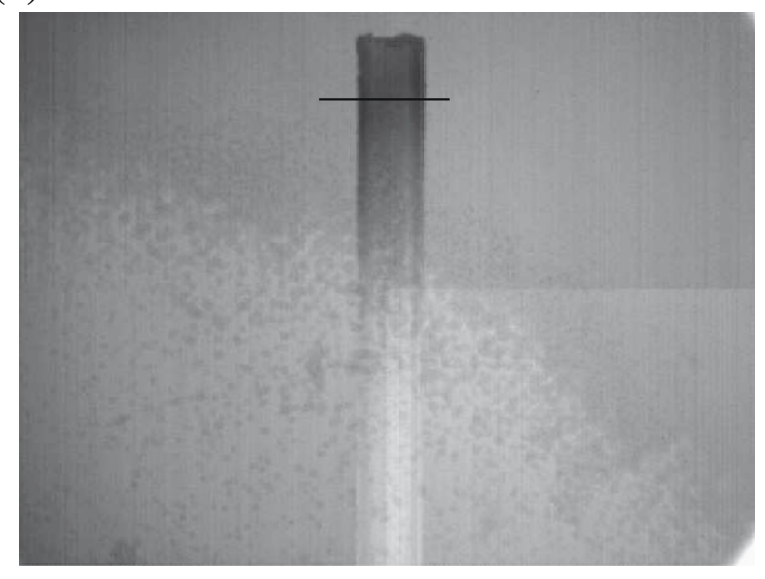

(b)

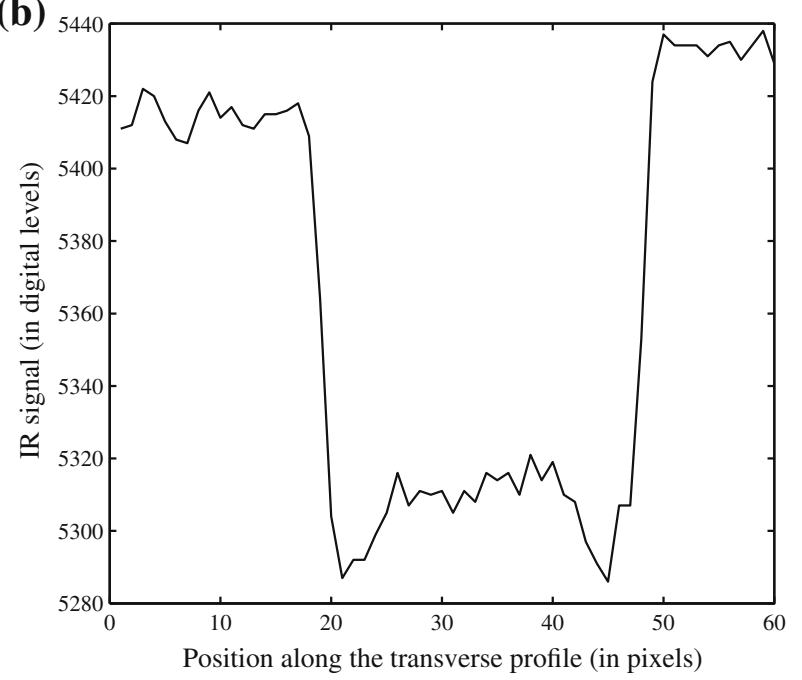

Fig. 3. (a) Raw IR image, at the beginning of an evaporation experiment (the bulk meniscus is located near the tube entrance). The grayscale used makes the cool regions to appear darker. On this raw image, the noise caused by the unwanted fluxes and particularly the IR detector non-uniformity can be seen. The effect of the IR detector architecture (presence of 4 read-out registers separating the detector into 4 quadrants) and a vignette effect can notably be discerned. (b) IR signal (expressed in digital levels) along the transverse profile shown as a solid line in (a).

sidered to be constant on every transverse profile and is obtained by averaging the signal over the 10 central pixels (ie from pixels 28 to 38 for the transverse profile shown in Fig. 3b). The standard deviation is typically found to be around 5-10 digital levels, which gives an estimate of the temperature measurement uncertainty that is $\pm 0.05-0.1 \mathrm{~K}$. Longitudinal temperature profiles can be obtained by plotting the tube temperature (determined as just explained) as a function of the distance to the tube opened end.

Fig. 4a shows a typical raw IR image obtained at the end of an evaporation experiment. As the evaporation rate is then very low, no cooling effect can be straightforwardly detected in this noisy picture, contrary to Fig. 3a. Fig. 4b shows a longitudinal temperature profile, along the height of the region of interest (ROI) highlighted by the solid rectangle in Fig. 4 a (signal averaging being performed along the width of the ROI, as said before). Fig. 4c shows the same image as in Fig. 4a, after subtraction of the image at ambient temperature and Fig. $4 \mathrm{~d}$ the same signal profile as in Fig. 4b. On this signal profile, a temperature minimum, associated with the cooling due to evaporation, is clearly discernible and can be easily located. Its amplitude is -30 digital levels, that is roughly $-0.3 \mathrm{~K}$ compared to the ambient temperature and its location is 145 pixels from the top of the ROI. This slightly cooled region can also be seen in Fig. 4c, where it appears lighter. The magnitude and location of this cooling effect cannot be obtained using the temperature profile shown in Fig. $4 \mathrm{~b}$ as it is "masked" by the effect of unwanted fluxes, such as the Narcissus effect, and the non-uniform IR detector response. Detection of the temperature minimum location and magnitude on raw IR images, without subtraction of the image at ambient temperature, will lead to erroneous results as soon as the signal variation due to the cooling to be detected is of the same order of magnitude than that due to the unwanted flux and detector non-uniformity effects, which can be estimated to be approximately 50 digital levels (which corresponds to $\approx 0.5 \mathrm{~K}$ ). Fig. 4 illustrates the ability of the present IR image processing, based on an image subtraction technique associated with a pixel by pixel calibration, to detect very small cooling of tube wall. After careful inspection of some typical IR images, it is estimated that cooling as small as $0.05 \mathrm{~K}$ can be detected.

The above procedure is also applied to a circular tube (one half of the external diameter was then painted in black). Due to the curvature of the tube external wall, the signal is not constant along a transverse profile. Nevertheless, as the depth of field of the IR optics is larger than the tube external half diameter, the IR signal is measured properly, i.e. without signal lowering, along such a transverse profile. Also, the signal minimum, expressed in digital levels on the IR images, can be detected with confidence. It must be mentioned that converting this signal back to temperature (which was not necessary for the present study) would imply to consider the paint being diffuse and to correct the measurement for tube roundness.

\section{Experimental results}

In this section, some experimental results obtained using the experimental set-up and techniques detailed above are presented. Typical evolutions of the bulk meniscus position $z_{0}$ as a function of time, in a capillary tube positioned either vertically or horizontally, are shown in Fig. 5. When the tube is positioned horizontally (crosses in Fig. 5), the bulk meniscus position $z_{0}$ evolves linearly with time: the evaporation rate, which is proportional to $d z_{0} / d t$, is therefore constant (note the position $z=0$ corresponds to the top of the tube and thus $z_{0}$ is increasing when the bulk meniscus recedes into the tube). When the tube is positioned vertically (dots in Fig. 5), two distinct periods are seen. First, as long as $z_{0}<18 \mathrm{~mm}$, the evaporation rate is roughly constant and slightly lower than that measured in the horizontal case. Then, for $z_{0}>18 \mathrm{~mm}$, the evaporation rate decreases suddenly and then keeps on decreasing as time goes on. To illustrate the increase in the evaporation rate induced by the presence of the corner films in a capillary tube of square cross section, the evolution of $z_{0}$ measured for hexane evaporating from a circular capillary tube of $1 \mathrm{~mm}$ internal diameter, under the same conditions as for the square tube, is also plotted in Fig. 5 (circles). As can be seen from Fig. 5, the presence of the corner films considerably accelerates the evaporation whatever the tube orientation is, as expected. It also must be noticed that the evaporation kinetic measurement for the circular cross section tube is in very good agreement with the classical analysis of evaporation in a circular tube, often referred to as the Stefan diffusion tube problem [11]. The corresponding theoretical prediction is shown as a solid line in Fig. 5 and corresponds to a temporal evolution of $z_{0}$ as [11]:

$z_{0}=\sqrt{\frac{2 D M P_{a}}{\rho_{l} N_{a} k_{b} T_{a}} \ln \left(\frac{P_{a}-P_{\infty}}{P_{a}-P_{s}}\right) t}$ 
(a)

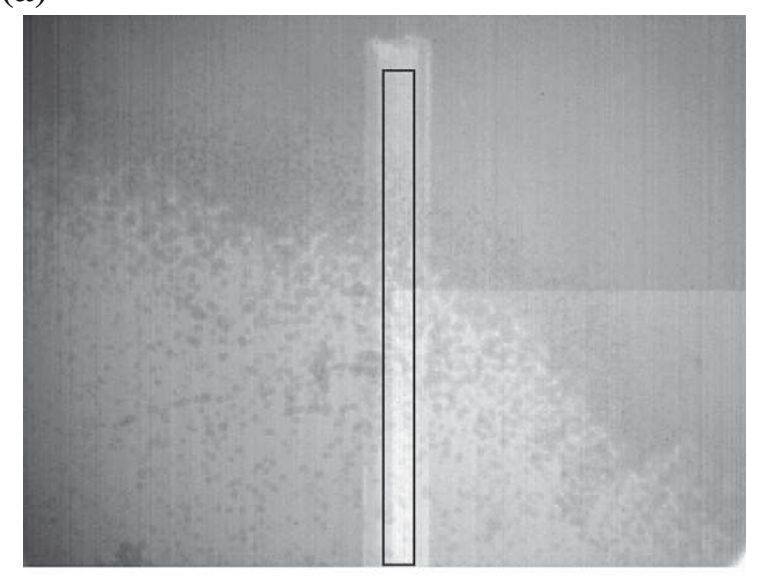

(c)

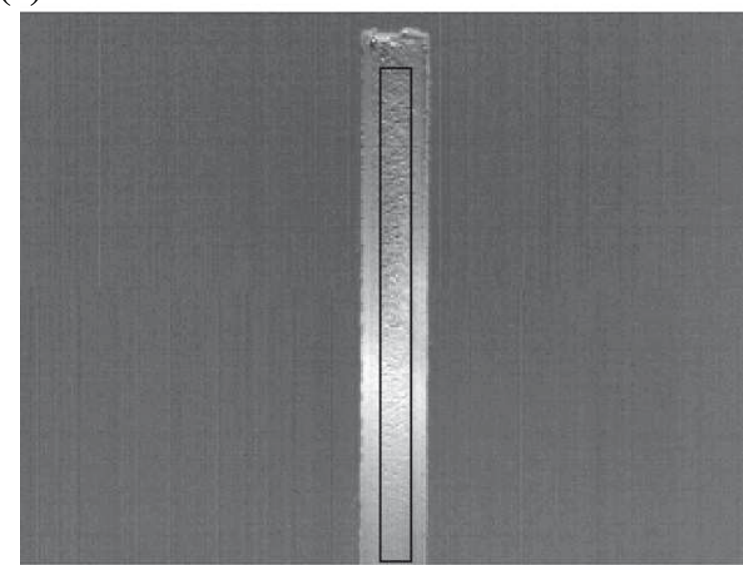

(b)

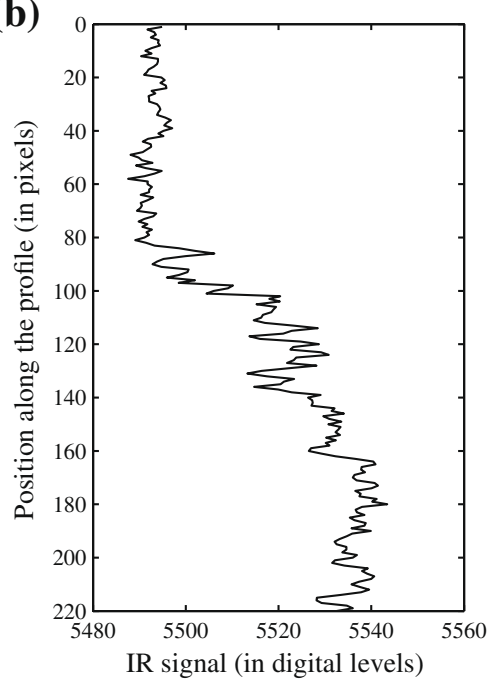

(d)

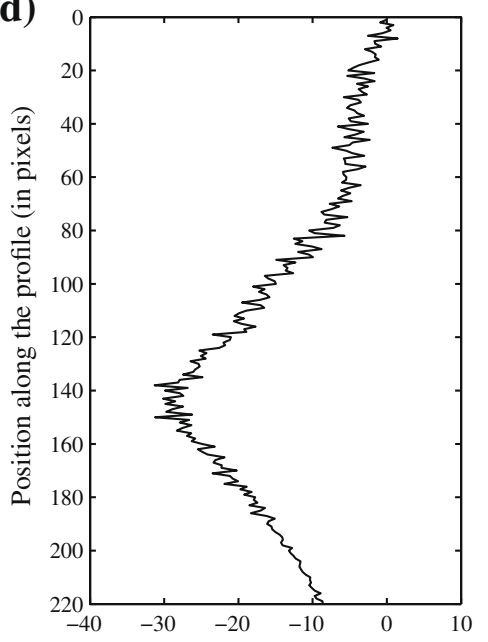

IR signal after image subtraction (in digital levels)

Fig. 4. (a) Raw IR image, at the end of an evaporation experiment. On this image, the noise caused by the unwanted fluxes and particularly the IR detector non-uniformity can be seen more clearly than in Fig. 3a. The effect of the IR detector architecture (presence of 4 read-out registers separating the detector into 4 quadrants) and a vignette effect can notably be discerned. (b) Longitudinal profile of the IR signal (expressed in digital levels) averaged along the width of the ROI shown as a solid rectangle in (a). (c) Same IR image than in Fig. 4, at the end of an evaporation experiment, after subtraction of an image taken at ambient temperature, when the tube is empty. (d) Longitudinal profile of the IR signal (expressed in digital levels) averaged along the width of the ROI shown as a solid rectangle in (c).

where $D=0.081 \mathrm{~cm}^{2} \mathrm{~s}^{-1}$ is the diffusion coefficient of hexane in air [17], $M=86.18 \mathrm{~g} \mathrm{~mol}^{-1}$ the molecular weight of hexane, $\rho_{l}=660.6$ $\mathrm{kg} \mathrm{m}^{-3}$ the density of liquid hexane, $N_{a}$ the Avogadro constant, $k_{b}$ the Boltzmann constant, $T_{a}=296 \mathrm{~K}$ the ambient temperature, $P_{a}=1 \mathrm{bar}$ the atmospheric pressure, $P_{\infty}=0$ the partial pressure of hexane at the tube exit and $P_{s}=0.185$ bar the saturation partial pressure of hexane at ambient pressure and temperature $\left(T_{a}, P_{a}\right)[18]$.

As explained in the previous section, the IR thermography technique allows to measure the temperature profile along the external wall of the capillary tube. Several longitudinal temperature profiles corresponding to various positions of the bulk meniscus are shown in Fig. 6, for the case of the square tube held vertically. The existence of a minimum for the temperature evolution along each profile is clearly displayed. This minimum is induced by the cooling sink effect due to evaporation. Noting that the evaporation process is slow and the tube wall relatively thin, the assumption that the minimum temperature location corresponds to the location where most of the phase change takes place within the tube will be made in the following, as in [12]. To support this assumption, the characteristic time for heat conduction within the tube wall can be eval- uated and shown to be much shorter than a characteristic time for evaporation. Borosilicate thermal diffusivity is $O\left(10^{-7}\right) \mathrm{m}^{2} \mathrm{~s}^{-1}$. For a wall tube thickness of $0.2 \mathrm{~mm}$, the characteristic time for heat diffusion within the tube wall is $\tau_{d} \approx 0.4 \mathrm{~s}$. This time is very short compared to a characteristic time for evaporation $\tau_{c}$ which can be taken as $\tau_{c}=d /\left(d z_{0} / d t\right)$, where $d$ is the tube internal side length ( $d=1 \mathrm{~mm}$ ) and $d z_{0} / d t$ the velocity at which the main meniscus recedes during the experiment. This receding velocity is at most $0.015 \mathrm{~mm} \mathrm{~s}^{-1}$ in the present experiments (see Fig. 5) and therefore $\tau_{c} \approx 65 \mathrm{~s} \gg \tau_{d}$.

Fig. 7a shows the evolution of the minimum temperature location, $z_{\min }$, as a function of $z_{0}$, in the case of a circular tube. The minimum temperature position corresponds almost perfectly with the receding meniscus position as expected for a circular tube. This confirms that the phase change location can be directly associated with the minimum temperature position, as already shown in [12]. The $\approx 0.5 \mathrm{~mm}$ constant offset is due to the fact that the minimum temperature is necessarily positioned slightly above the position of the bulk meniscus $z_{0}$, which is defined here as the most advanced point of the gas phase into the tube. 


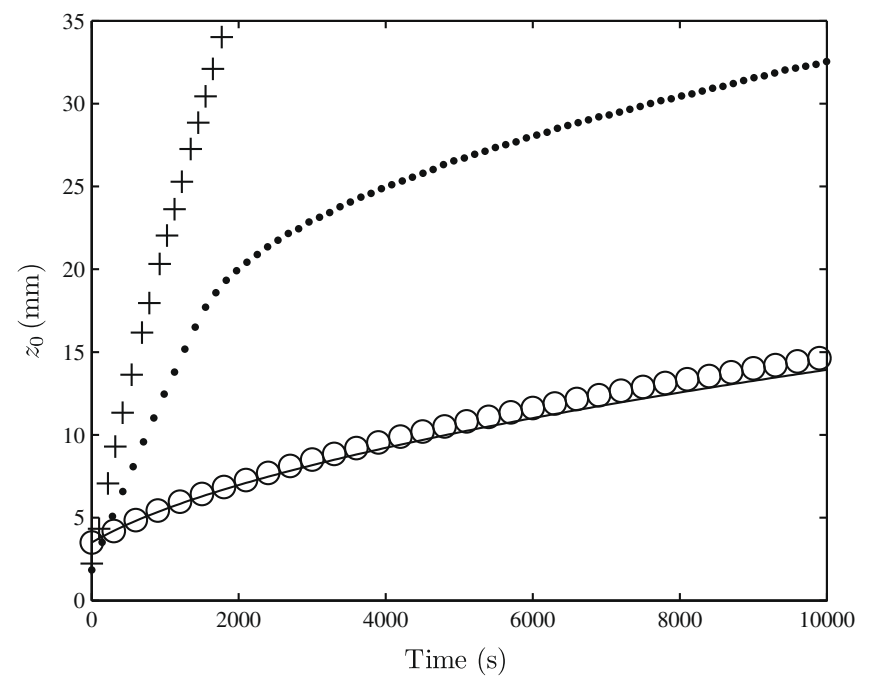

Fig. 5. Bulk meniscus position $z_{0}$ as a function of time. Crosses: tube positioned horizontally; dots: tube positioned vertically. The solid line corresponds to the theoretical prediction for a circular tube whereas the circle symbols are the corresponding experimental results.

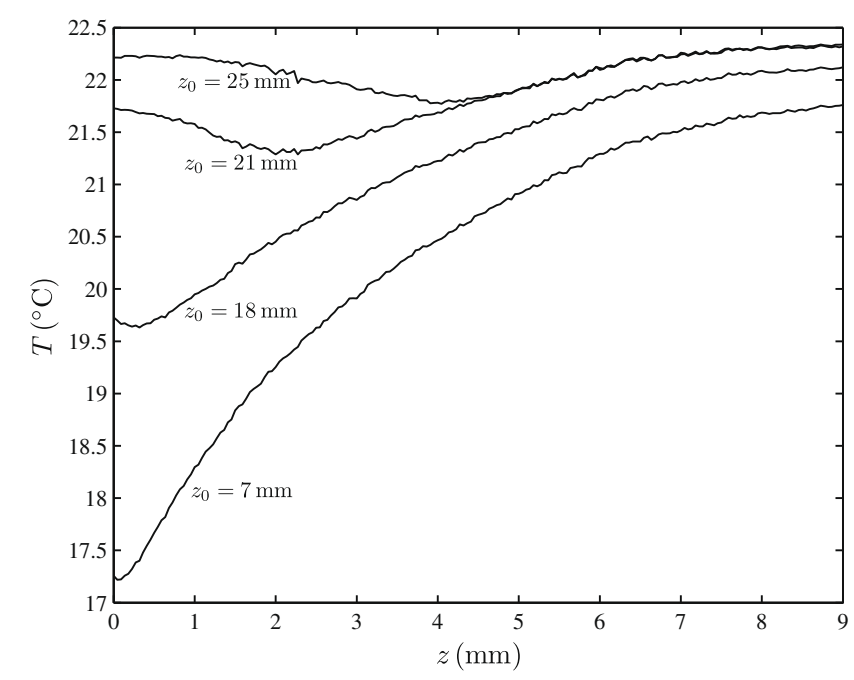

Fig. 6. Temperature profiles along the tube wall for various positions of bulk meniscus $z_{0}$, for the case of the square tube held vertically.

The evolution of the minimum temperature location for the square tube is now discussed. Typical evolutions of $z_{\min }$ as a function of $z_{0}$, in a tube positioned either vertically or horizontally, are shown in Fig. 7b. The evolution of $z_{\min }$ as a function of $z_{0}$ is different from the circular tube case shown in Fig. 7a and now depends on the tube orientation. In the horizontal case (crosses in Fig. 7b), the minimum temperature position remains constant and very close to the tube entrance over the whole range of bulk meniscus position investigated. This contrasts sharply with what is observed when the tube is positioned vertically (dots in Fig. 7b). In this case, as long as $z_{0}<18 \mathrm{~mm}, z_{\min }$ is located at the tube entrance $\left(z_{\min } \approx 0\right)$, as when the tube is held horizontally. As soon as $z_{0} \geqslant 18 \mathrm{~mm}$, the tube wall temperature minimum location $z_{\text {min }}$ starts receding within the tube, increasing quasi-linearly with $z_{0}$. Together with Fig. 5, Fig. 7b illustrates the remarkable effects associated with the corner liquid films. The location of the phase change is not at the bulk meniscus in contrast with tubes of circular cross section and the evaporation rate is much greater owing to the capillary pumping through the corner films which results in a location of the phase change at the tube entrance or much closer to the tube entrance than for a circular tube (in the vertical case for $z_{0}>18 \mathrm{~mm}$ ).

The tube wall minimum temperature $T_{\min }$ as a function of $z_{0}$ is shown in Fig. 8. In the horizontal case (crosses in Fig. 8), $T_{\min }$ remains constant, $T_{\min } \approx 17.5^{\circ} \mathrm{C}$. In the vertical case, the value of $T_{\min }$ is similar to that measured for the horizontal case at the beginning of the experiment. Then, $T_{\min }$ begins to increase slightly until $z_{0} \approx 15 \mathrm{~mm}$. Consistently with the results shown in Figs. 5 and $7 \mathrm{~b}$, $T_{\min }$ increases rapidly when $z_{0} \approx 18 \mathrm{~mm}$. Over the last period after the rapid variation around $z_{0} \approx 18 \mathrm{~mm}, T_{\min }$ slowly tends toward the room temperature. These observations show that the information provided by the visualization and IR thermography are consistent: high cooling when evaporation is fast, low cooling when evaporation is slow.

All the previous results highlight the precious pieces of information about the evaporation process obtained by the combined use of infrared thermography and visualizations by ombroscopy. At this stage, we are left with an explanation for the difference observed between the horizontal and vertical cases. This is the subject of the next section.

\section{Discussion}

Qualitatively, the interpretation of the experimental results is as follows. In the horizontal case, it is conjectured that the thickness of the corner film remains essentially constant whatever the position of the bulk meniscus within the tube is. As a result, the phase distribution at the tube entrance does not change significantly as the bulk meniscus recedes. Since the evaporation rate is controlled by what happens at the tube entrance region, a constant evaporation rate must follow from the absence of significant evolution of the phase distribution, which is in agreement with the experimental results, see Fig. 5. This picture is also consistent with the fact that the minimum temperature location remains stuck at the tube entrance and that the value of this minimum temperature remains constant, see Fig. 7b and Fig. 8. In agreement with the results presented in [9] for a case where the evaporation cooling effect is negligible, it is conjectured that, in the vertical case, the effect of gravity leads to a progressive thinning of the films as the bulk meniscus recedes into the tube and therefore to a variation of the evaporation rate (Fig. 5). The abrupt change in Figs. 5, $7 \mathrm{~b}$ and Fig. 8 around $z_{0} \approx 18 \mathrm{~mm}$ should correspond to the moment when the tip of the corner films begins to recede inside the tube. Also, the evaporation rate significant decrease for $z_{0}>18 \mathrm{~mm}$ reported in Fig. 5 is consistent with the fact that the receding of film tip implies the occurrence of an additional mass transfer resistance corresponding to the diffusive transport in gas phase from the film tip to the tube entrance. The film tip is viewed here as the location where the phase change takes place. According to this analysis, the film tip location corresponds therefore to the temperature minimum location, $z_{\min }$, and the length of the corner film is given by $z_{0}-z_{\min }$.

This qualitative analysis can be supported using a simple model of flow within the films. Following Coquard et al. [7], the liquid flow rate within each of the four corner films can be expressed as:

$q(z)=-\rho_{l} \frac{A_{c} R^{2}}{\beta \mu_{l}}\left(\frac{d p_{l}}{d z}-\rho_{l} g\right)$ with $z_{f}<z<z_{0}$,

where $\mu_{l}$ is the liquid viscosity and $\beta$ is a dimensionless resistance depending on the shape of the corner and on the boundary condition at the liquid-air interface. For a free boundary condition at the liquid-gas interface, a perfectly sharp corner and a perfectly wetting liquid, $\beta=113$ [19]. The film tip location is noted $z_{f}$ and, according to the present analysis, corresponds to $z_{\min }$. In Eq. (8), 
(a)

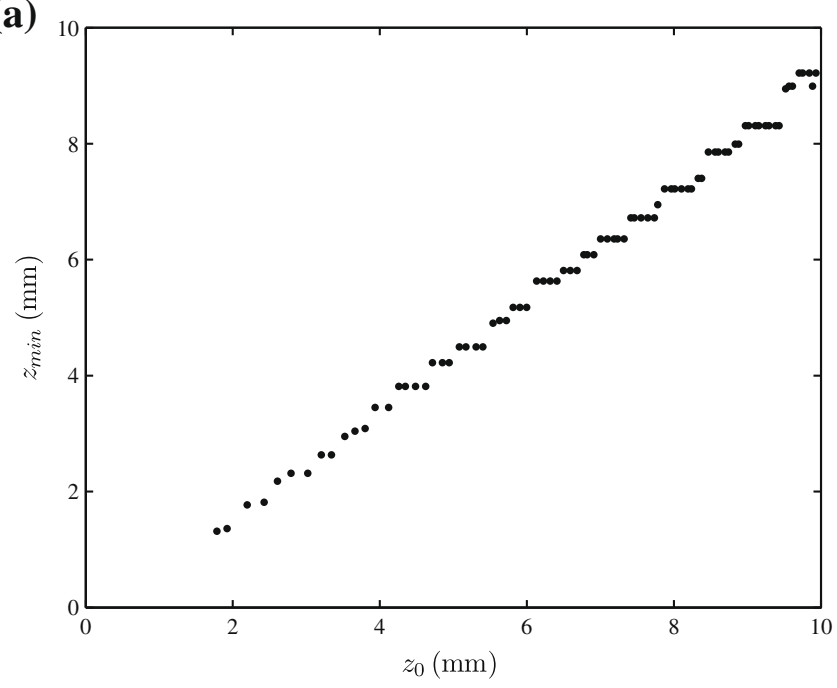

(b)

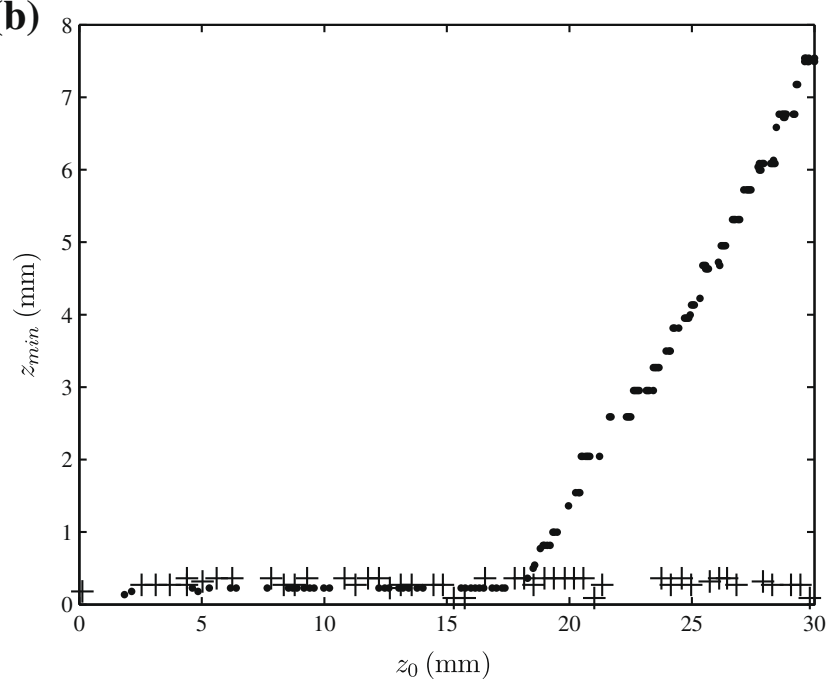

Fig. 7. (a) Evolution of the minimum temperature location along the capillary tube, $z_{\min }$, as a function of the bulk meniscus position, $z_{0}$, for a circular tube. (b) Evolution of the minimum temperature location along the capillary tube, $z_{\min }$, as a function of the bulk meniscus position, $z_{0}$, for a square tube. Crosses: tube positioned horizontally; dots: tube positioned vertically.

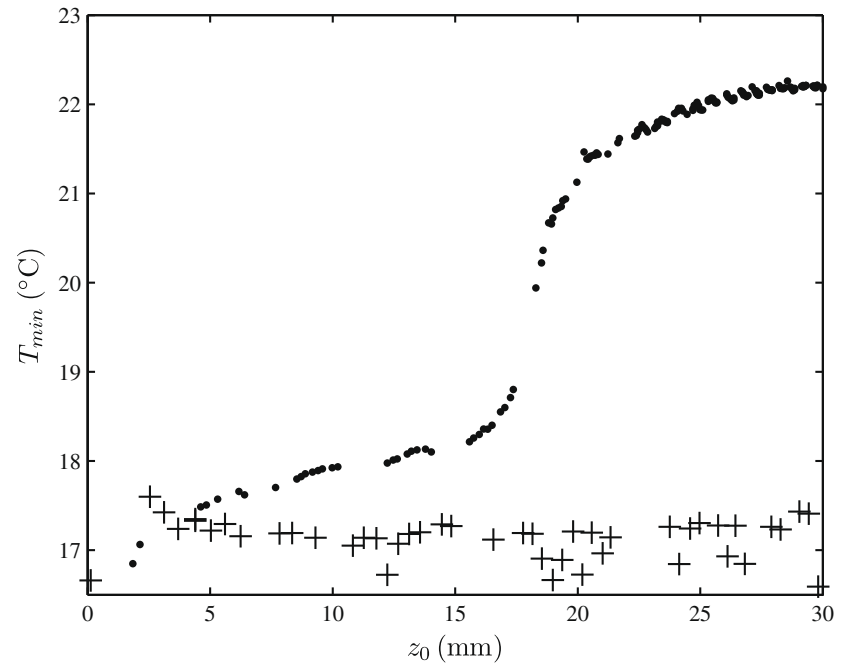

Fig. 8. Value of the temperature minimum along the capillary tube, $T_{\min }$, as a function of the bulk meniscus location, $z_{0}$. Crosses: tube positioned horizontally; dots: tube positioned vertically.

$A_{c}$ is the cross section area of the film at position $z$. It can be expressed as:

$A_{c}=\lambda R^{2}$,

where the geometrical factor $\lambda$ is given by $\lambda=\cos ^{2} \theta(\cos \theta+\sin \theta)-$ $\pi / 4+\theta$ where $\theta$ is the liquid contact angle [8]. As sketched in Fig. 9a, $R$ is the corner film meniscus curvature radius in the tube cross section plane and is a function of $z$. Note that the longitudinal curvature, i.e. the curvature along the tube axial direction $z$, is neglected in this simple model. The longitudinal curvature affects the shape of the elongated films very close to their tips, as can be checked on high magnification images of the films tips. Clearly, if the longitudinal curvature were to be non-negligible compared to the transverse one $(1 / R(z))$, it would be in this region of the films, which is of a very limited spatial extension. Thus, as far as the consequence on the description of the evaporation phenomenon is concerned, it can be expected that to take into account the longitudinal curvature would result only in a marginal correction to the case where only the transverse curvature is considered. Recently, Yang and Homsy [20] have studied the case of an elongated liquid film in a V-shaped wedge, with an imposed axial temperature gradient. The stationnary film shape is fixed by the competition between the Marangoni stress and the capillary pressure gradient. They have shown that to neglect the longitudinal curvature is justified over the full film extension, by deriving an expression for the capillary pressure drop and showing that it is dominated everywhere by the transverse curvature term compared to the axial one (note this remains true as long as the sum of the contact angle and of the corner half-angle is not $\pi / 2$, which is the case in the present study, where $\theta \approx 0$ and the half-angle is $\pi / 4$ ). In the present model, the film tip region is thus modelled as a region of sharp transition where the film thickness goes very abruptly from a finite positive value to zero, as sketched in Fig. 9b (see [8] for more details). The pressure in the corner film, $p_{l}$, is linked to the surface tension $\gamma$ and curvature of the liquid interface by Laplace's law. With the assumption of a negligible contribution of the curvature in the $z$ direction, this gives:

$p_{l}=P_{a}-\frac{\gamma}{R} \quad$ for $\quad z_{f}<z<z_{0}$.

Under the assumption that the shape of the bulk meniscus is essentially identical to its quasi-static shape, in the absence of gravity forces, we have for a perfectly wetting liquid:

$p_{l}\left(z_{0}\right)=P_{a}-\frac{3.77 \gamma}{d}$

where $d$ is the internal side length of the square tube [21]. Therefore $R=d / 3.77$ at $z=z_{0}$. Combining the previous equations leads to the following expression for the liquid flow rate in one corner:

$q(z)=-\rho_{l} \frac{\lambda}{\beta \mu_{l}}\left(\gamma R^{2} \frac{d R}{d z}-R^{4} \rho_{l} g\right) \quad$ with $\quad z_{f}<z<z_{0}$.

As shown in [6], the transport of liquid vapour by diffusion in the gas phase is only significant in the film tip region and can be neglected further below in the tube. As in [5] or [8], we assume therefore that the phase change takes place only at the tip of the films. Consequently, the flow rate $q$ does not change with $z$ and can be computed from the experimental data using the mass balance equation:

$4 q(z)=-\rho_{l}\left(d^{2}-4 A_{c}\left(z_{0}\right)\right) \frac{d z_{0}}{d t}$. 
(a)

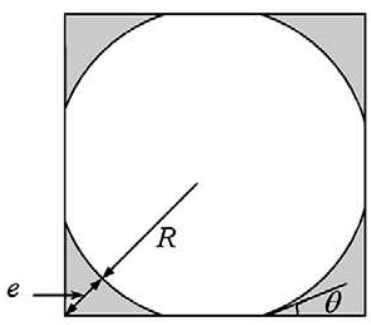

(b)

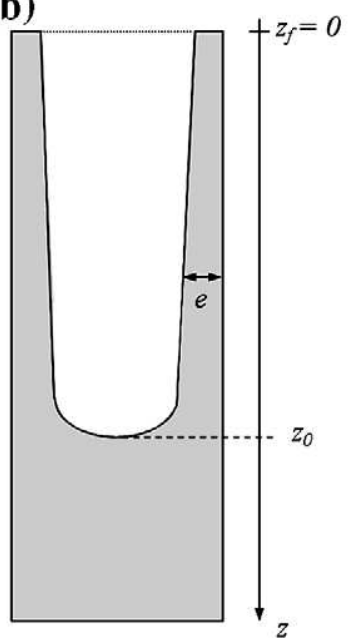

(c)

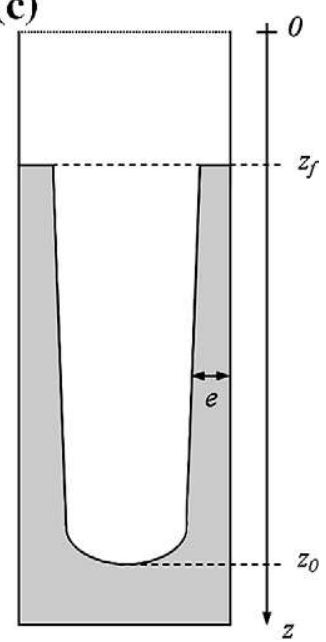

Fig. 9. (a) Radius of curvature $R$ and film thickness $e$ of the corner meniscus interface in the tube cross section plane. (b) and (c) Sketches of the liquid films tip shape used in the present modelling, respectively before and after the film depinning from the tube entrance.

Combining Eqs. (12) and (13) leads to:

$\left(1-\frac{4 \lambda}{(3.77)^{2}}\right) \frac{d z_{0}}{d t}=\frac{4 \lambda}{d^{2} \beta \mu_{l}}\left(\gamma R^{2} \frac{d R}{d z}-R^{4} \rho_{l} g\right)$ with $z_{f}<z<z_{0}$

Using the tube internal side length $d$ as a characteristic length scale, the problem governing the evolution of $R$ can be finally expressed in dimensionless form as:

$\frac{R^{\prime 2}}{1+(\mathrm{Bo} / \mathrm{Ca}) R^{\prime 4}} \frac{d R^{\prime}}{d z^{\prime}}=\mathrm{Ca}$ with $R^{\prime}=1 / 3.77$ at $z^{\prime}=z_{0}^{\prime}$.

Two dimensionless numbers appears in the above equation. The capillary number $\mathrm{Ca}$, which characterizes the competition between the capillary and viscous effects, is defined by:

$\mathrm{Ca}=\frac{\mu_{l} \beta\left(1-\frac{4 \lambda}{3.77^{2}}\right) \frac{d z_{0}}{d t}}{4 \lambda \gamma}$,

whereas, the Bond number Bo, which characterizes the competition between the capillary and gravity effects is defined by:

Bo $=\frac{\rho_{l} g d^{2}}{\gamma}$.

Typical values of capillary and Bond numbers are available in Table 1. Integrating Eq. (15) from $z_{f}^{\prime}$ to $z_{0}^{\prime}$ gives (omitting the superscript ' $)$ :

$F\left((\mathrm{Bo} / \mathrm{Ca})^{1 / 4} R\left(z_{f}\right)\right)-F\left((\mathrm{Bo} / \mathrm{Ca})^{1 / 4} R\left(z_{0}\right)\right)+\mathrm{Ca}^{1 / 4} \mathrm{Bo}^{3 / 4}\left(z_{0}-z_{f}\right)=0$,

where

Table 1

Values of capillary and bond numbers. For the vertical tube case, the maximum and minimum values correspond to the two distinct periods seen in Fig. 7, and are obtained respectively before and after the depinning of the corner film from the top of the capillary tube.

\begin{tabular}{lll}
\hline & Bo & Ca $(\min -\max )$ \\
\hline Horizontal & 0 & $\approx 4 \times 10^{-5}$ \\
Vertical & 0.36 & $1.76 \times 10^{-6}-2.13 \times 10^{-5}$ \\
\hline
\end{tabular}

$$
\begin{aligned}
F(X)= & \frac{\sqrt{2}}{8} \ln \left(\frac{X^{2}-X \sqrt{2}+1}{X^{2}+X \sqrt{2}+1}\right)+\frac{\sqrt{2}}{4} \operatorname{atan}(X \sqrt{2}+1) \\
& +\frac{\sqrt{2}}{4} \operatorname{atan}(X \sqrt{2}-1) .
\end{aligned}
$$

Using as inputs $d z_{0} / d t, z_{0}$ and $z_{f}$ (i.e. $z_{\min }$ ) deduced from the experimental data, the implicit Eq. (18) can be solved numerically to determine the evolution of $R\left(z_{f}\right)$, the radius of curvature $R$ at the film tip. Note that $R\left(z_{f}\right)$ is directly related to the film thickness in the film tip region. Indeed, under the assumptions of the present simplified model, the thickness $e$ of the corner film (in the intersection between the corner angle bisector plane and the tube cross section plane) can be estimated as $e=(\sqrt{2} \cos \theta-1) R$, see Fig. 9a.

The evolution of $R\left(z_{f}\right)$ as a function of $z_{0}$ for the vertical case is shown as crosses in Fig. 10. The evolution of $R\left(z_{f}\right)$ obtained assuming a purely hydrostatic equilibrium within the film is also shown (solid line in Fig. 10). In this case, the pressure gradient due to the flow is neglected and $d p_{l} / d z=\rho_{l} g$, so that $\gamma d R / d z=R^{2} \rho_{l}$ g according to Eq. 12. In dimensionless form, $d R^{\prime} / d z^{\prime}=R^{\prime 2}$ Bo, so that:

$\operatorname{Bo}\left(z_{0}^{\prime}-z_{f}^{\prime}\right)=\frac{1}{R^{\prime}\left(z_{f}^{\prime}\right)}-\frac{1}{R^{\prime}\left(z_{0}^{\prime}\right)}$.

As can be seen from Fig. 10, $R\left(z_{f}\right)$ is found to decrease significantly with $z_{0}$, as expected. In contrast, the same procedure applied to the horizontal case, i.e. taking $g=0$, leads to negligible variations of $R\left(z_{f}\right)$, i.e. $R\left(z_{f}\right) \approx R\left(z_{0}\right)$, in agreement with the analysis of the experimental data proposed at the beginning of this section.

Interestingly, Fig. 10 shows that the thinning of the film is essentially due to gravity effects at the beginning of evaporation since the evolutions of $R\left(z_{f}\right)$ given by Eqs. (18) and (20) are very close for small $z_{0}$. Then, viscous effects become increasingly important and contribute to the film thinning in addition to gravity effects. This is clearly seen from the increasing discrepancy between the purely hydrostatic evolution of $R\left(z_{f}\right)$ (solid line) and the evolution including the viscous effects (crosses in Fig. 10), at least up to $z_{0} \approx 18 \mathrm{~mm}$. This is explained as follows. First, we note that the evaporation rate is essentially constant for $z_{0}<18 \mathrm{~mm}$ (see Fig. 5). At the very beginning of the evaporation experiment, the cross section area of the film, which varies as $R^{2}$ according to Eq. 9, is the largest and the velocity induced within the film is not sufficient to generate a significant pressure gradient, due to viscous effects, compared to the hydrostatic one. Then, as the film cross section area diminishes, this velocity increases. As a result 


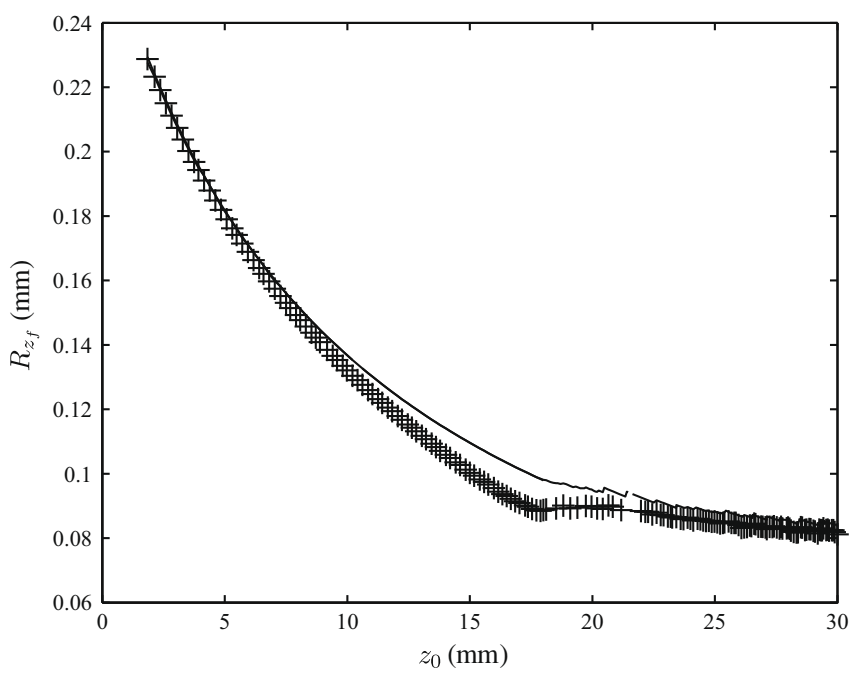

Fig. 10. Radius of curvature at the top of the film, $R_{z_{f}}$ as a function of the bulk meniscus position $z_{0}$. Crosses: capillary-viscous-gravity analysis. Solid line: hydrostatic analysis. Note that the film thickness $e$ is given by $e=(\sqrt{2} \cos \theta-1) R \approx 0.41 R$ for $\theta \approx 0^{\circ}$, as is the case in the present study.

the pressure gradient due to viscous effects increases and eventually becomes non-negligible compared to the hydrostatic pressure gradient. This contributes in turn to thin the film further (from Eq. 12 , the pressure gradient induced by viscous effects roughly scales as $\left.q / R^{4}\right)$. As can be seen from Fig. 10, the film tip thickness decrease is significantly slower for $z_{0}>18 \mathrm{~mm}$, i.e. when the film tip recedes into the tube. Owing to the additional mass transfer resistance due to diffusion in gas phase between the film tip and the tube entrance, the flow rate within the film diminishes. As a result, the pressure gradient generated by viscous effects tends to decrease and the discrepancy between the purely hydrostatic model and the model including the viscous effects tends to reduce. However, the viscous effects are crucial to limit the extension of corner films since according to Eq. (20), $1 / R\left(z_{f}\right)$ diverges with $z_{f}-z_{0}$ in the purely hydrostatic case.

We conclude that the model used here is fully consistent with the analysis of the present experimental data presented at the beginning of this section. One question, however, is why the thickness of the film is not zero at the receding film tip but remains positive (it is roughly equal to $37 \mu \mathrm{m}$, which corresponds to a corner film meniscus radius of curvature of $90 \mu \mathrm{m}$, see Fig. 10). Physically, we expect that the film completely dries out (regardless of possible adsorbed films) when the bulk meniscus has sufficiently receded into the tube. The explanation could be due to the fact that the model considered here is based on some too crude assumptions in the film tip region, which is essentially modelled as a discontinuity region between the film and the dry zone developing in the entrance region of the tube when the tip film recedes inside the tube, see Fig. 9c. More refined models would imply a much more detailed analysis of liquid flow and diffusive transport in the film tip region as well as the consideration of temperature variations. For example, it was shown in [12] that Marangoni convection may exist in the liquid right below the meniscus in a circular tube. Although the impact of the Marangoni convection on the overall evaporation rate is unclear, Marangoni convection may also affect the transfers in corner film tip region.

However, a simpler explanation can be proposed. Regardless of the complexity of the transport mechanisms details in the film tip region, the results presented in the present paper suggest that the introduction of a cut-off film thickness within the framework of the present model would provide an attractive and simple approach to the modelling of evaporation in tubes of polygonal cross section.

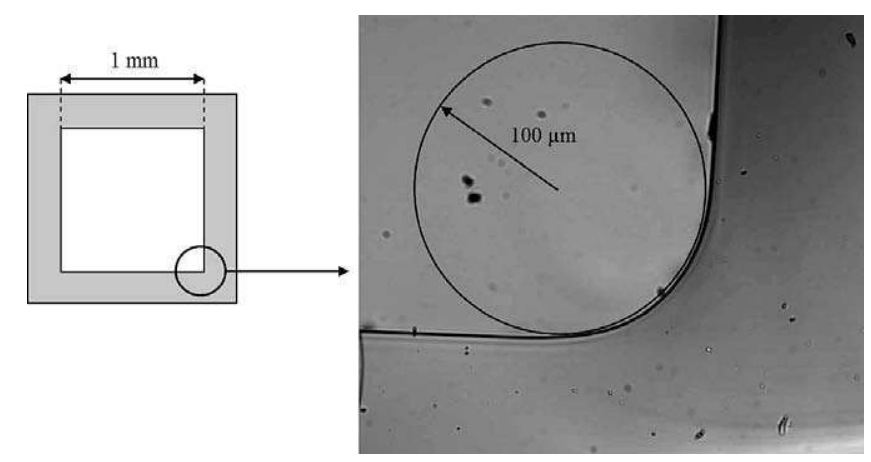

Fig. 11. Roundness of the square capillary tube internal corner, as visualized by an optical microscope with a $20 \times$ magnification. Rough measurements of the corner roundness over the tube four corners lead to a mean value of $100 \mu \mathrm{m}$ over the 4 corners with a standard deviation lower than $10 \mu \mathrm{m}$.

In fact, contrary to what is assumed in the modelling, the tube internal corner is not perfectly sharp for the square tube used in the experiments. As shown in Fig. 11, visualizations at high magnification of the tube corner show that the corner is in fact rounded with an internal curvature radius of about $100 \mu \mathrm{m}$. This introduces a geometrical cut-off film curvature radius, which is fully consistent with the results reported in Fig. 10. It is should be pointed out, however, that using the cut-off thickness introduced by the degree of roundedness of the tube internal corners together with the simple model presented in this paper only represents a simplified approach, which has the advantage to be semi-analytical and can be sufficient for qualitative parametric studies or, as here, which can be used as a tool to interpret experimental results. As briefly described in [9], accounting properly for the corner internal roundness makes the modelling significantly more involved since the corner degree of roundedness non linearly affects the hydraulic conductivity of the films [19]. In particular, this implies to develop a numerical approach for solving the flow problem within the films in conjunction with a proper accounting of the coupled heat and mass transfers controlling the evaporation rate. This is left for a future work.

\section{Conclusion}

In the present work, the use of an IR thermography technique, coupled with visualizations by ombroscopy, enabled us to highlight the dynamic of thick liquid corner films as well as their impact on the evaporation process. A specific infrared thermography data processing based on the proper consideration of all important radiative fluxes has been developed in order to measure the temperature of the capillary tube external wall with a good accuracy. This permits to track the bulk meniscus position evolution as well as the evolution of the tube wall temperature minimum, i.e. the films tip position. The dynamic of the film is controlled by the competition between the capillary, gravity and viscous effects. When the tube is horizontal (no gravity effects), the evaporation rate is constant and the films extend up to the entrance of the tube in accordance with the predictions for the "capillary regime" presented in previous works [6,7]. When the tube is vertical, the films remain attached to the tube entrance in a first phase and the evaporation rate is comparable to the horizontal case. In a second period, the films tip recedes into the tube and the evaporation rate slows down significantly. A simple analysis based on a previous model, [8], shows that this is due to the progressive thinning of the film under the action of both gravity and viscous effects. Our results are consistent with previous modelling attempts [6-8]. However they suggest that a more refined modelling is needed to obtain good quantitative predictions of evaporation from a single polygonal tube. In particular, the roundness of 
the tube internal corners must be incorporated in the analysis. It was pointed out in [8] that somewhat tiny details of the tube geometry can be of great effect on the evaporation process. The results presented here confirm this experimentally. Although evidenced in this paper for a single tube, this should be also true for more complex systems such as a microfluidic system made of interconnected channels or a porous medium.

\section{Acknowledgment}

Financial support from GIP ANR “Intensifilm” (Project ANR-06BLAN-0119-01) is gratefully acknowledged.

\section{References}

[1] M. Prat, Recent advances in pore-scale models for drying of porous media, Chem. Eng. J. 86 (1-2) (2002) 153-164.

[2] S. Jin, K.S. Breuer, Diffusion-limited evaporation in long microchannels, in: Proceedings of ASME International Mechanical Engineering Congress \& Exposition 2003 (IMECE03), Washington, DC, USA.

[3] B. Camassel, Contribution à l'étude du changement de phase liquide vapeur en milieu poreux, in: Ph.D. Thesis, Institut National Polytechnique de Toulouse, 2003.

[4] J.C.T. Eijkel, B. Dan, H.W. Reemeijer, D.C. Hermes, J.G. Bomer, A. van den Berg, Strongly accelerated and humidity independent drying of nanochannels induced by sharp corners, Phys. Rev. Lett. 95 (2005). 256107-1/4.

[5] A.G. Yiotis, A.G. Boudouvis, A.K. Stubos, I.N. Tsimpanogiannis, Y.C. Yortsos, The effect of liquid films on the drying of porous media, AIChE J. 50 (11) (2004) 2721-2737.

[6] B. Camassel, N. Sghaier, M. Prat, S. Ben Nasrallah, Ions transport during evaporation in capillary tubes of polygonal cross section, Chem. Eng. Sci. 60 (2005) 815-826.
[7] T. Coquard, B. Camassel, M. Prat, Evaporation in capillary tubes of square cross section, in: Proceedings of ASME Heat Transfer Conference 2005 (HT2005), San Francisco, CA, USA.

[8] M. Prat, On the influence of pore shape, contact angle and film flows on drying of capillary porous media, Int. J. Heat Mass Transfer 50 (2007) 1455-1468.

[9] F. Chauvet, P. Duru, S. Geoffroy, M. Prat, Three periods of drying of a single square capillary tube, Phys. Rev. Lett. 103 (2009) 124502.

[10] F. Chauvet, P. Duru, M. Prat, Study of evaporation in capillary tubes by infrared thermography and ombroscopy technique, in: Proceedings of ASME International Conference on Nanochannels, Microchannels and Minichannels 2008 (ICNMM08), Darmstadt, Germany.

[11] R.B. Bird, W.E. Stewart, E.N. Lightfoot, Transport Phenomena, Wiley, New York, 2002.

[12] C. Buffone, K. Sefiane, IR measurements of interfacial temperature during phase change in a confined environment, Exp. Therm. Fluid Sci. 29 (2004) 6574.

[13] N. Kim, Analyse expérimentale d'un jet turbulent impactant sur une plaque plane et sur un obstacle de section carrée, in: Ph.D. Thesis, Université Paul Sabatier, 2005.

[14] N. Horny, FPA camera standardisation, Infrared Phys. Technol. 44 (2003) 109119.

[15] M.F. Westby, Heat transfer measurements using infrared thermography in rarefied flows, Technical Memorandum W1, Defence Research Agency, Farnborough, UK, 1992.

[16] D. Pajani, Thermographie IR: prudence pour mesurer la température des fils chauds, vol. 778, 2005, Available from <www.mesures.com>.

[17] R.C. Reid, J.M. Prausnitz, B.E. Poling, The Properties of Gases \& Liquids, fourth ed., McGrawHill, New York, 1987.

[18] Chemistry WebBook, National Institute of Standards and Technology, Available from <http://webbook.nist.gov/chemistry/>.

[19] D. Zhou, M. Blunt, F.M. Orr Jr., Hydrocarbon drainage along corners of noncircular capillaries, J. Colloid Interf. Sci. 187 (1997) 11-21.

[20] Li Yang, G.M. Homsy, Steady three-dimensional thermocapillary flows and dryout inside a V-shaped wedge, Phys. Fluids 18 (2006) 042107.

[21] H. Wong, S. Morris, C. Radke, Three dimensional menisci in polygonal capillaries, J. Colloid Interf. Sci. 148 (2) (1992) 317-336. 